\title{
The pgm locus and pigmentation phenotype in Yersinia pestis
}

\author{
Tereza Cristina Leal-Balbino ${ }^{1}$, Nilma Cintra Leal ${ }^{1}$, Mirna Gisele Medeiros do Nascimento ${ }^{1}$, \\ Maria Betânia Melo de Oliveira ${ }^{1}$, Valdir de Queiroz Balbino ${ }^{2}$ and Alzira Maria Paiva de Almeida ${ }^{1}$ \\ ${ }^{I}$ Fundação Oswaldo Cruz, Centro de Pesquisas Aggeu Magalhães, Departamento de Microbiologia, \\ Recife, PE, Brazil. \\ ${ }^{2}$ Universidade Federal de Pernambuco, Departamento de Genética, Recife, PE, Brazil.
}

\begin{abstract}
The pigmentation (pgm) locus is a large unstable area of the Yersinia pestis chromosome composed of a segment of iron acquisition (HPI) linked to a pigmentation segment. In this work we examined the mobility of HPI and the pigmentation segment in three $Y$. pestis isolates using successive subcultures on Congo red agar (CRA) plates. Strain P. CE 882 was shown to be highly stable while strains $P$. Exu 340 and P. Peru 375 dissociated into several phenotypes, PCR analysis showing evidence of changes in the pgm locus of the derived cultures. Strains P. Exu 340 and P. Peru 375 produced previously unreported cultures positive for the pesticin/yersiniabactin outer membrane receptor $(p s n+)$ but negative for the iron-regulated protein (irp2-), suggesting the occurrence of rearrangements in this chromosomal region and either a sequential loss or the loss of separated segments. These results provide evidence that besides deletion en bloc, specific rearrangements are also involved in the deletion events for that locus.
\end{abstract}

Key words: Y. pestis, high pathogenicity island, pgm, psn.

Received: August 24, 2004; Accepted: May 10, 2005.

\section{Introduction}

The genus Yersinia (Enterobacteriaceae) contains 11 species, three of which, pestis (causing bubonic plague) and the enteropathogens pseudotuberculosis and enterocolitica, are pathogenic in humans (Perry and Fetherston, 1997), with plague strains as well as enterocolitica biotype 1B and pseudotuberculosis serogroups I and III also being highly pathogenic in mice (Carniel, 2001). All pathogenic strains of Yersinia carry the conserved 70-kb plasmid associated with Yersinia virulence (pYV) which is essential for virulence, with $Y$. pestis also harboring two other plasmids (pFra and pPst) that are necessary for the expression of complete virulence in this organism (Perry and Fetherston, 1997).

The chromosome of highly pathogenic yersiniae contains a region involved in siderophore-mediated iron acquisition, which is considered a high-pathogenicity island (HPI) (Carniel, 2001). The $Y$. pestis HPI is located in a large unstable area of chromosomal DNA (102-kb) first identified by Fetherston et al. (1992) and named the pigmentation locus (pgm). This area is composed of a $\sim 35 \mathrm{~kb}$ iron acqui-

Send correspondence to Tereza Cristina Leal-Balbino. Fundação Oswaldo Cruz, Centro de Pesquisas Aggeu Magalhães, Departamento de Microbiologia, Campus da Universidade Federal de Pernambuco s/n, Cidade Universitária, 50670-420 Recife, PE, Brazil. E-mail: cristina@cpqam.fiocruz.br. sition segment (the HPI) linked to a $\sim 68 \mathrm{~kb}$ pigmentation segment (Buchrieser et al., 1998). In the Y. pestis iron acquisition segment there is a cluster of genes formed by the iron-regulated protein (irp), yersiniabactin siderophore biosynthetic protein $(y b t)$ and the pesticin/yersiniabactin outer membrane receptor ( $p s n$ ) cluster (called the $p s n$ cluster in $Y$. pestis or the pesticin/yersiniabactin receptor protein $(f y u A)$ cluster in $Y$. enterocolitica) which is involved in the biosynthesis of the yersiniae-siderophore (yersiniabactin or $\mathrm{Ybt}$ ). Besides having two different names ( $p s n$ and $f y u A$ ), this gene cluster has dual functions in that it encodes for both the pesticin bacteriocin receptor and $\mathrm{Ybt}$ (Carniel, 2001; Rakin et al., 1994, 1996). The hemin storage $(h m s)$ locus is located in the pigmentation segment and is responsible for the Congo red binding pigmented phenotype $(\mathrm{pgm}+)$ of colonies grown on Congo red-agar plates (CRA) (Buchrieser et al., 1998; Fetherston e Perry, 1994; Pendrak and Perry, 1993).

In $Y$. pestis, the iron acquisition and transport systems are also under the regulation of a ferric uptake regulation (Fur) protein encoded by the fur gene located in another area of the chromosome outside the iron acquisition segment (Staggs and Perry, 1992).

Two copies of the insertion sequence (IS) IS 100 flank the $102-\mathrm{kb}$ region of $Y$. pestis. Homologous recombination between two IS100 sequences leads to the deletion of the 
segment responsible for the pigmentation (Pgm-) phenotype (Fetherston et al., 1992). Pgm- cultures are avirulent in mice inoculated via the peripheral route, except when coadministered with iron (Fetherston et al., 1992; Jackson and Burrows, 1956). These deletions were believed to occur en bloc. However, the existence of Pgm- irp $2+$ cultures (Iteman et al., 1993) suggests that the pigmentation segment is mobile and independent of the iron acquisition segment. Buchrieser et al. (1998) identified several rearrangements involved in different phenotypes of colonies developed on CRA plates from cultures of different geographical origin.

The aim of the work described in this paper was to determine the mobility of HPI and the pigmentation segment in Y. pestis isolates through successive subcultures on CRA plates and using PCR for the determination of the genes from the two segments of the pigmentation locus and fur.

\section{Material and Methods}

\section{Bacterial isolates and culture conditions}

The study involved three $Y$. pestis isolates: an early isolate (P. Exu 340) derived from the bone marrow of a finger from a fatal human case of plague in 1969; a more recent Brazilian isolate (P. CE 882) derived from a blood culture from a plague case in 1997 in the Brazilian State of Ceará; and a 1994 isolate (P. Peru 375) from a Peruvian plague patient. The Girard-Robic EV76 vaccinal strain served as the pigmentation phenotype control. These strains are held at the culture collection of the Department of Microbiology, CPqAM (Leal-Balbino et al., 2004). For this work, the parent cultures were reactivated by growing them at $28{ }^{\circ} \mathrm{C}$ in brain heart infusion (BHI) broth, (Difco, USA) for $24 \mathrm{~h}$ and then plating on blood agar base (BAB; Difco, USA) for $48 \mathrm{~h}$.

After reactivation, the cultures were submitted to successive subculturing on Congo red agar (CRA) plates incubated at $26{ }^{\circ} \mathrm{C}$ for 4 days (Surgalla et al., 1970) for phenotypic observation. There were five different types of colonies: red (R), white (W), half red/half white (RW), red with a thin white border (RWB) and white with a red pin-point center (WRP). Phenotypically selected colonies from the CRA plates were grown in brain heart infusion (BHI) broth and their genomic DNA extracted according to Maniatis et al. (1982) for PCR assay.

The number of subcultures and colonies analyzed varied for each parent culture, the derivative strains were allocated a strain number commencing with YP (for $Y$. pestis) followed by the parent strain number, subculture number and phenotype (Figure 1).

\section{PCR analysis}

Parent and derivative cultures of strains P. CE 882, P. Exu 340 and P. Peru 375 were analyzed for the presence of genes from the iron acquisition ( $p s n, y b t E$, irp2) and the pigmentation segments $(h m s \mathrm{H})$ using PCR with primers derived from published sequences (Rakin and Heeseman, 1995; Buchrieser et al. 1999; Guilvout et al. 1993; Schubert et al. 1998).

To further understand the events involved in the modifications of the pgm locus, the presence of the ansP gene, located in the central area of this locus (between the iron ac-

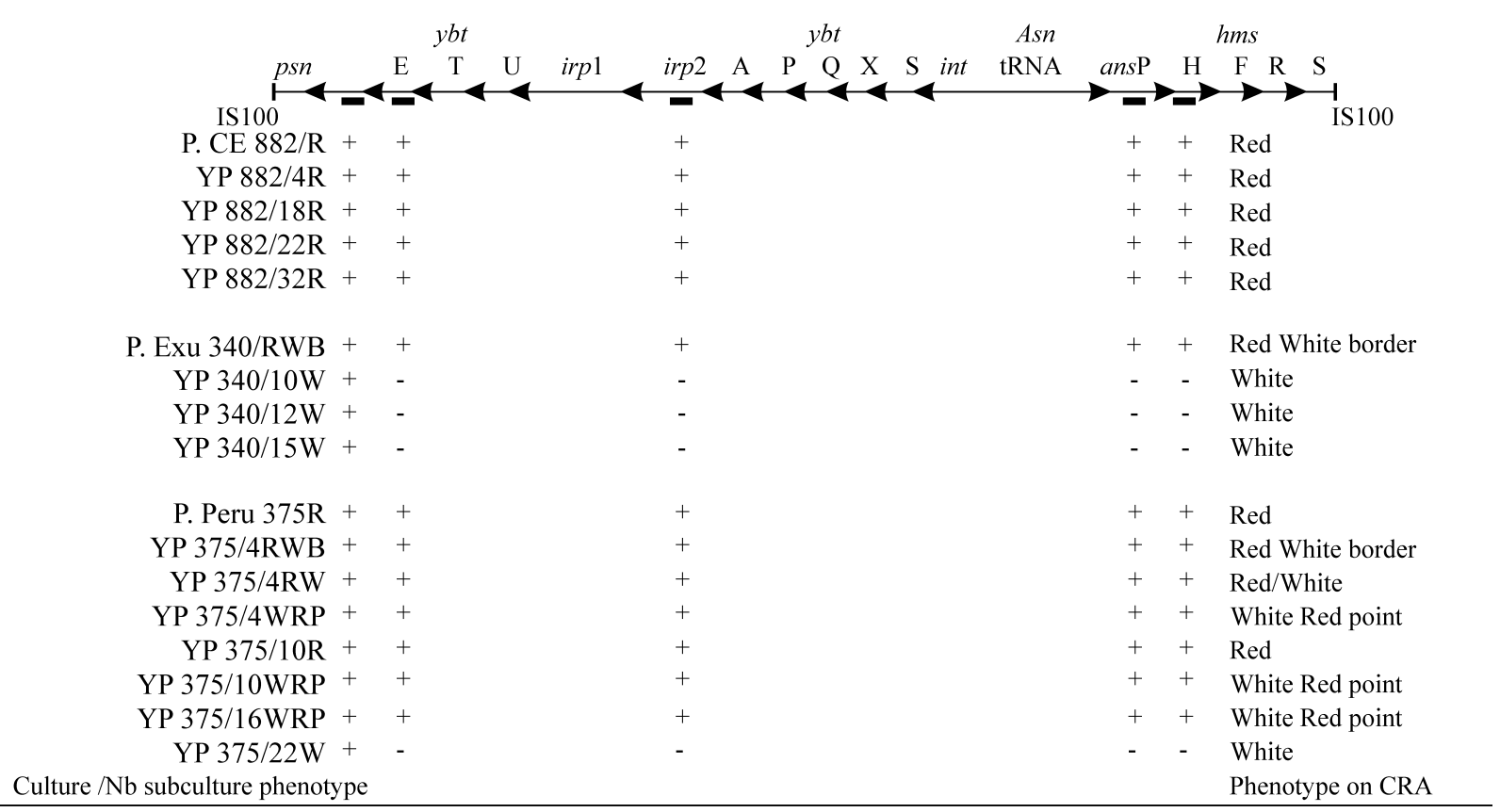

Figure 1 - Schematic representation of the pgm locus of Yersinia pestis showing the results of PCR amplification of segments located in the high-pathogenicity island (HPI) and in the pigmentation segment of the different strains. The bars below the $p g m$ locus represent the targeted genes. $(+)=$ presence of the amplified DNA segment; $(-)=$ no amplified segment. 
quisition and the pigmentation segments), was investigated with specific PCR primers obtained using the 'PrimerSelect' program (DNAstar, Inc.).

The role of Fur in the different phenotypes obtained on CRA plates from the parent cultures was examined by determining the presence of the fur gene by PCR using primers derived from the published sequence of this gene (Hinnebush et al. 1998).

The PCR reactions for $p s n, y b t E$, irp2, hms $\mathrm{H}$, fur and L-asparagine permease (ans $P$ ) genes, consisted of $20 \mathrm{ng}$ of strain DNA, 1U of Taq DNA polymerase (CENBIOT, BR), $160 \mu \mathrm{M}$ of each dNTP (Amersham Biosciences of Brazil Ltda, BR), $1.5 \mathrm{mM} \mathrm{MgCl}_{2}, 20$ pmol of each primer, $50 \mathrm{mM}$ $\mathrm{KCl}$ and $10 \mathrm{mM}$ Tris-HCl-pH 8 in a final volume of $25 \mu \mathrm{L}$. Amplifications were performed in a thermocycler (Perkin Elmer, USA) programmed for 25 cycles of $1 \mathrm{~min}$ at $94{ }^{\circ} \mathrm{C}$, $2 \mathrm{~min}$ at the annealing temperatures (Tm) and $3 \mathrm{~min}$ at $72{ }^{\circ} \mathrm{C}$, finishing with a final elongation step of $7 \mathrm{~min}$ at $72{ }^{\circ} \mathrm{C}$. The sequences of the forward primer (FP), reverse primer (RP) the size of the expected segments and Tm are shown in Table 1. The annealing regions of the primers on the targeted genes (pgm locus) were confirmed using the 'PrimerSelect' program.

\section{Construction of the $\mathrm{HmsH}$ probe and hybridization}

The presence of the pigmentation segment was determined in the RWB and WRP phenotypes by amplification of the $h m s \mathrm{H}$ gene and the three phenotypes ( $\mathrm{R}, \mathrm{RWB}$ and WRP) were compared with regard to this genomic region. Total DNA of the parent strains (P. Exu 340, P. CE 882 and P. Peru 375) and derived cultures was digested with the DraI and $A v a \mathrm{I}$ restriction enzymes and hybridized with a probe directed to the hms $\mathrm{H}$ gene.

The HmsH probe was obtained by PCR and labeled during the amplification reaction using the DIG-dNTP DNA Labeling and Detection Kit (Boehringer Mannheim, DE) following the manufacturer's instructions.

Based on the restriction map of the $h m s \mathrm{H}$ gene obtained with the 'PrimerSelect' program we chose DraI and AvaI to digest the total DNA extracted from the $Y$. pestis cultures. The restriction fragments were separated by electrophoresis, transferred by vacuum to nylon membranes using the VacuGeneTMXL (Amersham Biosciences of Brazil
Ltda, BR) system and hybridized with the HmsH probe. Pre-hybridization (for $30 \mathrm{~min}$ ) and overnight hybridization were performed at $65^{\circ} \mathrm{C}$ in a hybridization oven (ColeParmer, USA) following the protocol provided with the kit (Amersham Biosciences of Brazil Ltda, BR).

\section{Analysis of the psn gene sequence}

To confirm that the segment amplified by PCR was really part of the psn gene, the amplified psn segments of the $Y$. pestis cultures were eluted from the gel, purified using the Concert Nucleic Acid Purification Kit (Gibco, USA) and analyzed in an ABI 310 automatic sequencer (Applied Biosystems, USA). The nucleotide sequences obtained were analyzed with the BLAST program (www. ncbi.nlm.nih.gov) (Altschul et al., 1997).

To compare the segments of the psn gene amplified from the different cultures, the PCR products were digested with EcoRV and BclI and the fragments separated by agarose gel electrophoresis.

\section{Nucleotide sequence accession number}

The Genbank accession number for the sequence of the psn gene of strain YP 340/15W is AY461411.

\section{Results and Discussion}

Pigmentation analysis of the colonies grown on CRA plates revealed important differences among the three isolates studied. Strain P. CE 882 has proved to be highly stable, since its isolation in 1997 (Leal and Almeida, 1999) it has produced only red ( $\mathrm{R}$ or $\mathrm{Pgm}+$ ) colonies on CRA plates and no phenotypic alteration has been detected through 32 subcultures (Figure 1).

Strain P. Exu 340 was found to be unstable because when it was obtained in 1969 and first analyzed it was Pgm + but developed red colonies with a thin white border (RWB). After 10 subcultures of the RWB colonies only white (W or Pgm-) colonies were produced on CRA plates (Figure 1).

Strain P. Peru 375 also proved to be unstable, with its initially red colonies producing five different types of subculture colonies (R, W, RW and WRP). After the $22^{\text {nd }}$ subculture from RWB or WRP, completely white colonies were observed (Figure 1).

Table 1 - Primers used in the PCR analysis.

\begin{tabular}{|c|c|c|c|c|}
\hline Gene & Forward primer & Reverse primer & Size $(b p)$ & Annealing temperature $\left({ }^{\circ} \mathrm{C}\right)$ \\
\hline $\operatorname{irp} 2$ & 5'-ATTTCTGGCGCACCATCT-3' & 5'-GCGCCGGGTATTACGGACTTC-3' & 1000 & 50 \\
\hline hmsH & 5'-TAAAGAAAGACCCCACCAATC-3' & 5'-ATCATCGGCATCAAGCAAATC-3' & 730 & 55 \\
\hline ans $P$ & 5'-GCCGCTATTGCCCTGTTTTTGAT-3' & 5'-ACCGGGCATTTTGAAGGAGACAG-3' & 730 & 50 \\
\hline$y b t E$ & 5' - CCCTTACCCATTGCCGAAC-3' & 5'-TCCCCACCTCATCCAGCC-3' & 1200 & 50 \\
\hline fur & 5'-GAAGTGTTGCAAAATCCTGCG-3' & 5'-AGTGACCGTATAAATACAGGC-3' & 328 & 65 \\
\hline psn & 5'-CAACATCGTCACCCAGCA-3' & 5'-CGCAGTAGGCACGATGTTGTA-3' & 920 & 65 \\
\hline
\end{tabular}


PCR using primers directed to genes of both segments of the pgm locus resulted in amplification of all the expected DNA segments of the correct sizes corresponding to the genes irp2 (1000 bp), ybtE (1200 bp) and $h m s \mathrm{H}$ (730 bp) in the parent strain P. CE 882 and its derivative cultures (Figures 1, 2B-D). There was also amplification of all the targeted genes of strain P. Exu 340 (RWB) and of the different P. Peru 375 phenotypes (R, RWB and WRP) analyzed. These genes were not amplified in the white cultures derived from strains P. Exu 340 and P. Peru 375 (Figure 1).

A segment of the expected size $(730 \mathrm{bp})$ for the ans $P$ gene was amplified in the parent strain P. CE 882 (R) and in the four $(\mathrm{Pgm}+)$ derivative cultures analyzed after the $4^{\text {th }}$, $18^{\text {th }}, 22^{\text {nd }}$ and $32^{\text {nd }}$ subcultures (Figures $1,2 \mathrm{~A}$ ). This segment was also amplified in cultures of P. Exu 340 (RWB), P. Peru $375(\mathrm{R})$ and in the phenotypes RWB and WRP, analyzed after the $4^{\text {th }}, 10^{\text {th }}$ or $16^{\text {th }}$ subcultures from P. Peru 375. However, there was no amplification in the three white cultures obtained (YP 340/10W, YP 340/12W, YP 340/15W), respectively from the $10^{\text {th }}, 12^{\text {th }}$, and $15^{\text {th }}$ subcultures of $\mathrm{P}$. Exu 340 and from the white culture (YP 375/22W) obtained from the $22^{\text {nd }}$ subculture of P. Peru 375 (Figure 1). These results suggest that the central region of the $\mathrm{pgm}$ locus is complete in the R, RWB and WRP phenotypes and is deleted in the $\mathrm{W}$ phenotype.

The PCR analysis showed that the pgm locus is similar in the R, RWB and WRP phenotypes. However, the

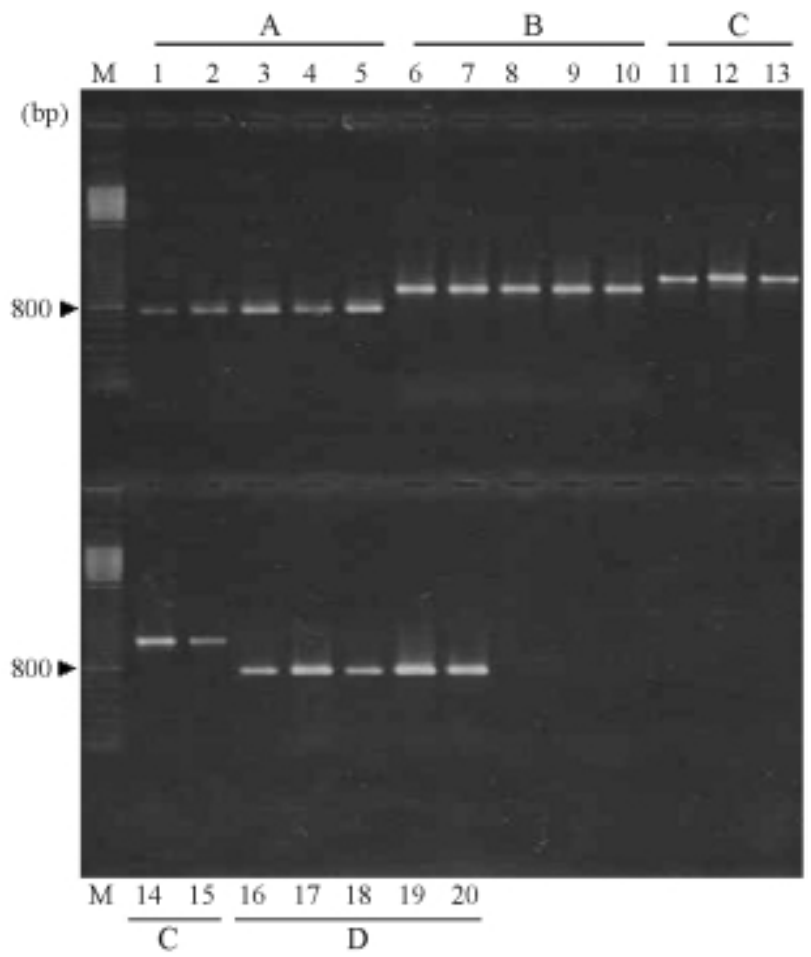

Figure 2 - PCR products from primers directed to the genes: ansp (A); irp 2 (B); $y b t \mathrm{E}(\mathrm{C})$ and $h m s \mathrm{H}(\mathrm{D})$ and the strains P. CE 882R (lines 1, 6, 11, 16); YP 882/4R (lines 2, 7, 12, 17); YP 882/18R (lines 3, 8, 13, 18); YP 882/22R (lines 4, 9, 14, 19); and YP 882/32R (lines 5, 10, 15, 20); $\mathrm{M}=100$ bp DNA ladder.

HmsH probe only recognized the R and RWB phenotypes and the hybridization profiles obtained in the two phenotypes were identical (Figure 3). Despite the phenotypic difference, no alteration was detected in the pgm locus of the $\mathrm{R}$ and RWB isolates using either PCR or hybridization with the probe. The WRP phenotype was not recognized by the probe in spite of PCR amplification of the $h m s \mathrm{H}$ gene. This phenotype is probably composed of a mixed population, where the number of Pgm+ cells is too small and the target is not detected by hybridization, although efficiently amplified by PCR.

The amplification product of the expected size for the fur gene ( $328 \mathrm{bp}$ ) was obtained in all the parent and derivative cultures studied, independent of whether the phenotype was R, RWB, WRP or W, suggesting that the phenotypic modifications are not related to this gene (Figure 4).

Amplification of a segment of the expected size (920 bp) for the psn gene was observed in all cultures of strains P. CE 882, P. Exu 340, P. Peru 375 and derivative cultures, independent of their phenotype on CRA plates. Unexpectedly, this segment was also amplified in the white cultures (W) derived from strains P. Exu 340 and P. Peru 375,

A

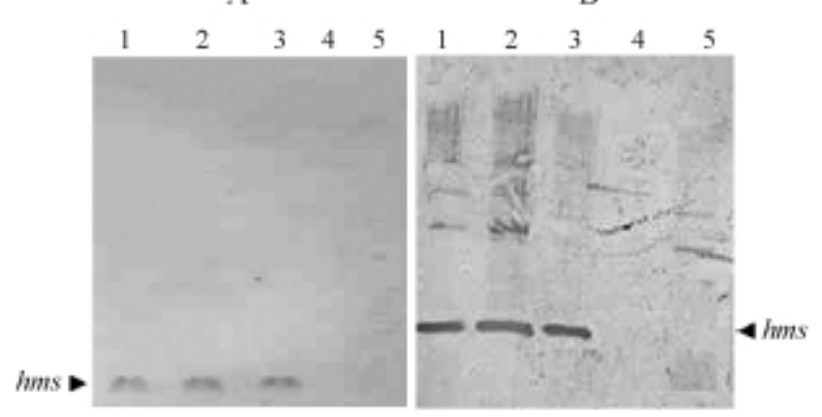

Figure 3 - Hybridization with the $\mathrm{HmsH}$ probe and DNA from strain $\mathrm{P}$. CE $882 \mathrm{R}$ as positive control (lines 1); P. Exu 340/RWB (line 2); YP 375/4RWB (line 3); YP 375/16WRP (line 4); and YP 375/22W (line 5), digested with $D r a \mathrm{I}(\mathrm{A})$ and $A v a \mathrm{I}(\mathrm{B})$.

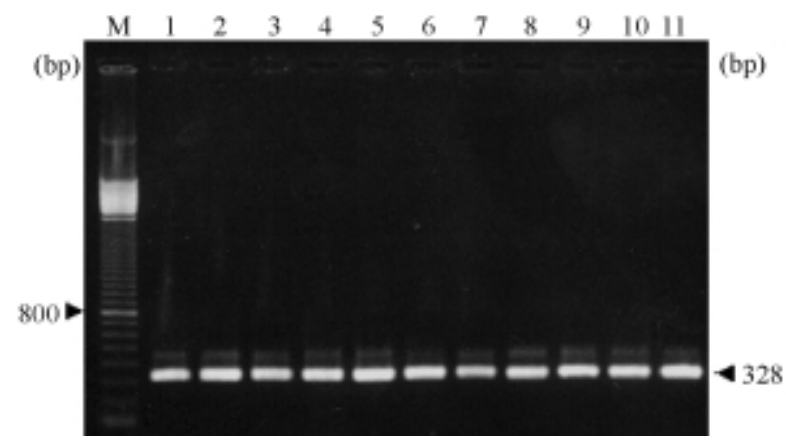

Figure 4 - PCR products from primers directed to the fur gene and strains P. CE 882R (line 1); YP 882/32R (line 2); P. Exu 340/RWB (line 3); YP 340/10W (line 4); YP 340/15W (line 5); P. Peru 375R (line 6); YP 375/4RW (line 7); YP 375/4RWB (line 8); YP 375/4WRP (line 9); YP 375/16WRP (line 10); YP 375/22W (line 11); $\mathrm{M}=100$ bp DNA ladder. 
in spite of the absence of the irp2, $y b t \mathrm{E}$, ans $\mathrm{P}$ and $h m s \mathrm{H}$ genes in these cultures (Figures 1, 5A).

Analysis of the results with the BLAST program revealed high homology between the $p s n$ gene of $Y$. pestis and the fyuA of $Y$. enterocolitica and $Y$. pseudotuberculosis and we also observed that the deduced sequence of the protein was similar to that of the FyuA protein of $Y$. enterocolitica, $Y$. pseudotuberculosis and E. coli. These results confirm the presence of the psn gene in the irp2- Pgm-Y. pestis cultures

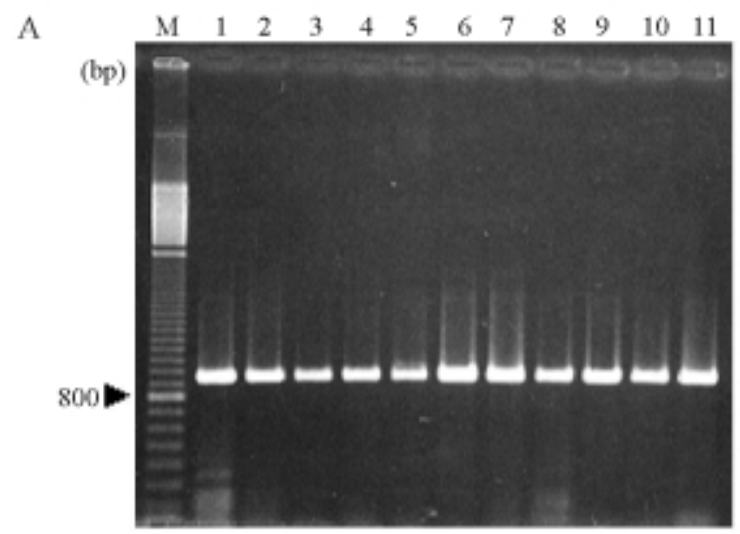

B

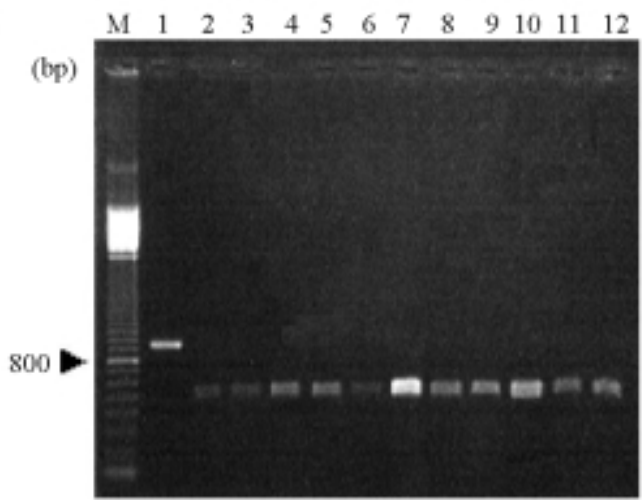

C

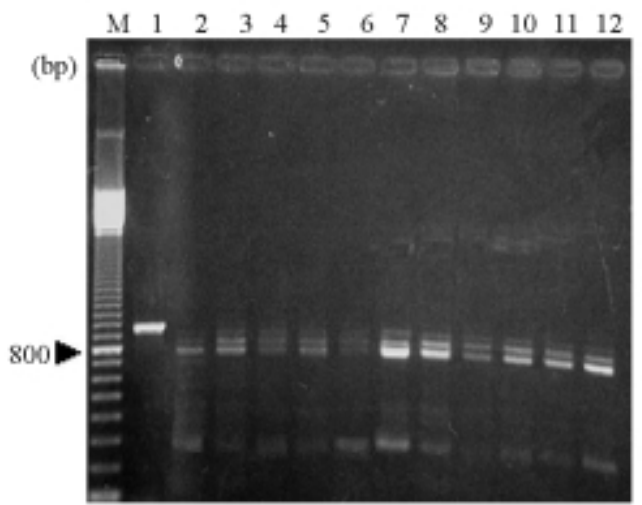

Figure 5 - PCR products from the primers directed to the gene $p s n(\mathrm{~A})$ and strains P. CE 882R (line 1); YP 882/32R (line 2); P. Exu 340/RWB (line 3); YP 340/10W (line 4); YP 340/15W (line 5); P. Peru 375R (line 6); YP 375/4RW (line 7); YP 375/4RWB (line 8); YP 375/4WRP (line 9); YP 375/16WRP (line 10); YP 375/22W (line 11); $\mathrm{M}=100$ bp DNA ladder; and digestion of the PCR products with $B c l \mathrm{I}(\mathrm{B})$ and $\operatorname{EcoRV}(\mathrm{C})$ : same cultures lines 2-12; undigested PCR from YP 882/32R: line 1; $\mathrm{M}=100 \mathrm{bp}$ DNA ladder. analyzed, and they also demonstrate high homology for the psn gene among yersiniae.

Comparison of agarose gel electrophoresis bands of the segments of the $p s n$ gene amplified in the different cultures and digested with $B c l$ I and $E c o$ RV revealed an identical restriction pattern in all the cultures (Figure 5B-C).

It has been reported that in $Y$. enterocolitica deletion of the fyuA irp2 genes occurs only in the fyuA part of the gene cluster. Three different types of cultures $(f y u \mathrm{~A}+$ irp2+, fyuA-irp2- and fyuA-irp2+) were observed in $Y$. enterocolitica by Rakin et al. (1994) but the psn + irp2- isolate found among the $Y$. pestis strains in the present study has not been previously described.

The irp1, irp2, fyuA fragment is unstable in $Y$. enterocolitica and $Y$. pseudotuberculosis. Lesic et al. (2004) showed that the excision of HPI in $Y$. pseudotuberculosis requires the combined actions of an integrase and a recombination directionality factor. In $Y$. enterocolitica, this instability is due to IS1328 or other insertion elements adjacent to fyuA (Fetherston et al., 1992; Rakin and Heeseman, 1995). The deletion may involve the $f y u \mathrm{~A}$ gene only or both $f y u \mathrm{~A}$ and irp2 (Rakin and Heeseman, 1995).

The finding of $p s n+i r p 2-$ cultures suggests the occurrence of rearrangements in this region and a sequential loss or the loss of separated segments. These events are also probably due to the presence of repetitive sequences (RS4 and RS5) or of insertion sequences (IS100) flanking the $Y$. pestis HPI (Fetherston et al., 1992; Buchrieser et al., 1998).

Our results suggest that the alteration of the $102-\mathrm{kb}$ region of $Y$. pestis involves not only deletion en bloc by homologue recombination between IS100 sequences flanking the region but also other specific rearrangements in this segment.

\section{Acknowledgments}

We thank Dr Osvaldo Pompílio (CPqAM/FIOCRUZ) for advice during the work. We are grateful to Silvana Santos and Yara Nakasawa for their technical assistance and the Brazilian Research Council Conselho Nacional de Desenvolvimento Científico e Tecnológico (CNPq) for financial support.

\section{References}

Altschul SF, Madden TL, Schaffer AA, Zhang J, Zhang Z, Miller W and Lipman DJ (1997) Gapped BLAST and PSI-BLAST: A new generation of protein database search programs. Nucleic Acids Res 25:3389-3402.

Buchrieser C, Prentice M and Carniel E (1998) The 102-kilobase unstable region of Yersinia pestis comprises a high-pathogenicity island linked to a pigmentation segment which undergoes internal rearrangement. J Bacteriol 180:2321-2329.

Buchrieser C, Rusniok C, Frangeul L, Couve E, Billault A, Kunst F, Carniel E and Glaser P (1999) The 102-kilobase pgm locus of Yersinia pestis: Sequence analysis and comparison of 
selected regions among different Yersinia pestis and Yersinia pseudotuberculosis strains. Infect Immun 67:4851-61.

Carniel E (2001) The Yersinia high-pathogenicity island: An iron-uptake island. Microbiol Infect 3:561-569.

Fetherston JD, Schuetze P and Perry RD (1992) Loss of the pigmentation phenotype in Yersinia pestis is due to the spontaneous deletion of $102 \mathrm{~kb}$ of chromosomal DNA which is flanked by a repetitive element. Mol Microbiol 6:26932704.

Fetherston JD and Perry RD (1994) The pigmentation locus of Yersinia pestis KIM6+ is flanked by an insertion sequence and includes the structural genes for pesticin sensivity and HMWP2. Mol Microbiol 3:697-708.

Guilvout I, Mercereau-Puijalon O, Bonnefoy S, Pugsley AP and Carniel E (1993) High-molecular-weight protein 2 of Yersinia enterocolitica is homologous to AngR of Vibrio anguillarum and belongs to a family of proteins involved in nonribosomal peptide synthesis. J Bacteriol 175:5488-5504.

Hinnebusch BJ, Gage KL and Schwan TG (1998) Estimation of vector infectivity rates for plague by means of a standard curve-based competitive polymerase chain reaction method to quantify Yersinia pestis in fleas. Am J Trop Med Hyg 58:562-569.

Iteman I, Guiyoule A, Almeida AMP, Guilvout I, Baranton G and Carniel E (1993) Relationship between loss of pigmentation and deletion of the chromosomal iron-regulated irp 2 gene in Yersinia pestis: Evidence for separate but related events. Infect Immun 61:2717-2722.

Jackson S and Burrows TW (1956) The virulence enhancing effect of iron on non-pigmented mutants of virulent strains of Pasteurella pestis. Brit J Exp Pathol 37:577-583.

Leal-Balbino TC, Leal NC, Lopes CV and Almeida AM (2004) Differences in the stability of the plasmids of Yersinia pestis cultures in vitro: Impact on virulence. Mem Inst Oswaldo Cruz 99:727-732.
Leal NC and Almeida AMP (1999) Diagnosis of plague and identification of virulence markers in Yersinia pestis by multiplex-PCR. Rev Inst Med Trop São Paulo 41:339-342.

Lesic B, Bach S, Ghigo J-M, Dobrindt U, Hacker J and Carniel E (2004) Excision of the high-pathogenicity island of Yersinia pseudotuberculosis requires the combined actions of this cognate integrase and Hef, a new recombination directionality factor. Mol Microbiol 52:1337-1348.

Maniatis T, Frisch E and Sambrook J (1982) Molecular Cloning: A Laboratory Manual. Cold Spring Harbor Laboratory, NY, pp 368-369.

Pendrak ML and Perry RD (1993) Proteins essential for expression of the Hms+ phenotype of Yersinia pestis. Mol Microbiol 8:857-864.

Perry RD and Fetherston JD (1997) Yersinia pestis - Etiologic agent of plague. Clin Microbiol Rev 10:35-66.

Rakin A, Saken E, Harmsen D and Heeseman J (1994) The pesticin receptor of Yersinia enterocolitica: A novel virulence factor with dual function. Mol Microbiol 13:253-263.

Rakin A and Heeseman J (1995) Virulence-associated fyuA/irp2 gene cluster of Yersinia enterocolitica biotype 1B carries a novel insertion sequence IS1328. FEMS Microbiol Lett 129:287-292.

Rakin A, Boolgakowa E and Heeseman J (1996) Structural and functional organization of the Yersinia pestis bacteriocin pesticin gene cluster. Microbiology 142:3415-3424.

Schubert S, Rakin A, Karch H, Carniel E and Heesemann J (1998) Prevalence of the "high-pathogenicity island" of Yersinia species among Escherichia coli strains that are pathogenic to humans. Infect Immun 66:480-5.

Staggs TM and Perry RD (1992) Fur regulation in Yersinia species. Mol Microbiol 6:2507-2516.

Surgalla MJ, Beesley ED and Albizo JM (1970) Pratical applications of new laboratory methods for plague investigations. Wld Hlth Org Bull 42:993-997.

Associate Editor: Sergio Olavo Pinto da Costa 